\title{
Synthesis and characterization of some novel coumarin based 2- pyridone heterocycles with their broad spectrum antimicrobial potency
}

\author{
Nisheeth C Desai* and Tushar J Karkar \\ (DST-FIST Sponsored \& UGC NON-SAP), Division of Medicinal Chemistry \\ Department of Chemistry, Mahatma Gandhi Campus \\ Maharaja Krishnakumarsinhji Bhavnagar University \\ Bhavnagar-364002, India \\ E-mail: dnisheeth@rediffmail.com \\ Tel. No. 0278-2439852
}

Keywords: Coumarin, 2-Pyridone, Antimicrobial activity, MIC

\begin{abstract}
The synthesis of a novel series of 6-((arylidene)amino)-2-oxo-1-((1-(2-oxo-2H-chromen3-yl)ethylidene)amino)-4-phenyl-1,2-dihydropyridine-3,5-dicarbonitriles 4a-o were synthesized and structures of compounds have been elucidated by IR, ${ }^{1} \mathrm{H}$ NMR, ${ }^{13} \mathrm{C}$ NMR and mass spectral data. Antimicrobial activity was measured against certain strains of bacteria and fungi by serial broth dilution. Evaluation of antimicrobial activity shown that the compounds $(\mathbf{4 g}, \mathbf{4 j}, \mathbf{4 k}$ and $\mathbf{4 0})$ were found to be most active against selected bacterial strains and compounds $(\mathbf{4 d}, \mathbf{4 m}$ and $\mathbf{4 n})$ were found to be most active against selected fungal strains.
\end{abstract}

\section{Introduction}

Coumarin and 2-pyridone derivatives play a vital role in the theoretical development of heterocyclic chemistry along with being used extensively in organic synthesis. The action of bacterial infections still remains a significant therapeutic problem. It led to develop infectious diseases and the increasing number of multidrug-resistant microbial pathogens. Therefor since the past few decades much attention has been given to the design and synthesis of new types of pharmacologically diverse structural hybrid molecules [1]. The present work is dealing with the synthesis of novel series of 6-((arylidene)amino)-2-oxo-1-((1-(2-oxo-2H-chromen-3-yl)ethylidene)amino)-4-phenyl1,2 dihydropyridine-3,5-dicarbonitriles 4a-o. It is well known that coumarin derivatives possess a wide range of medicinal indications, such as anthelmintic, anticoagulant, hypnotic and insecticidal properties [2]. The antimicrobial activity of coumarin derivatives is reported in the literature $[3,4,5,6,7]$. Potential of various natural and synthetic coumarin derivatives proved its importance as anti-inflammatory [8] antioxidant properties [9] anticancer [10,11] and monoamine oxidase inhibitors [12]. Novobiocin and chlorobiocin have reported as antimicrobials containing a coumarin skeleton [13].

Pyridone and their derivatives play a vital role in some biological processes and have significant chemical and pharmacological importance $[14,15,16]$. 2-Pyridones motifs found to possess useful pharmacological activities, such as analgesic [17], antimalarial [18], anti-HIV [19], phytotoxic [20], and antitumoral [21] properties. The versatility of 2-pyridone and its potential to yield derivatives with a wide range of biological activities has made it a useful structure for further molecular investigation. Moreover, 2-pyridones are a class of newly exposed potent antibacterial agents that are of specific concern due to their in vitro and in vivo antibacterial potencies against the bacterial type II DNA topoisomerases, which consist of two highly homologous enzymes-DNA gyrase and topoisomerase IV [22,23]. In accordance with our previous work [24,25,26] and medicinal importance of coumarin and 2-pyridone motifs we report herein the synthesis of a new class of 6((arylidene)amino)-2-oxo-1-((1-(2-oxo-2H-chromen-3-yl)ethylidene)amino)-4-phenyl-1,2dihydropyridine-3,5-dicarbonitriles $\mathbf{4 a - 0}$ as potential antimicrobial agents. 


\section{Results and discussion}

\subsection{Chemistry}

The synthetic route to the proposed compounds is shown in Scheme 1. The synthesis of 6-((arylidene)amino)-2-oxo-1-((1-(2-oxo-2H-chromen-3-yl)ethylidene)amino)-4phenyl-1,2-dihydropyridine-3,5-dicarbonitriles 4a-o have carried out as follows: 3-acetyl- $2 H$ chromen-2-one (1) was prepared by Salicylaldehyde (A) $(0.01 \mathrm{~mol})$ and equimolar amount of ethyl acetoacetate (B) in dry methanol using $1 \mathrm{~mL}$ piperidine as a catalyst. Reaction mixture was stirred at room temperature for $30 \mathrm{mins}$ and after the stirring, crystals were observed at the bottom of the RBF. In the first step, compound (2) was prepared by mixture of 3-acetyl- $2 H$-chromen-2-one $(0.01$ mol) (1) and 2-cyanoacetohydrazide $(0.01 \mathrm{~mol})$ in presence of methanol $(30 \mathrm{~mL})$ with catalytic amount of glacial acetic acid and refluxed at $50-60{ }^{\circ} \mathrm{C}$. In the second step, intermediate (2) (0.01 mol) was refluxed with 2-benzylidenemalononitrile (E) $(0.01 \mathrm{~mol})$ and catalytic amount of piperidine in presence of ethanol $(99 \%, 30 \mathrm{~mL})$ as a solvent gave compound (3). Intermediate (3) $(0.01 \mathrm{~mol})$ was further treated with various aromatic aldehydes $(0.01 \mathrm{~mol})$ and ethanol $(99 \%, 30$ $\mathrm{mL}$ ) refluxed and the solid separated to obtain novel compounds of the series 4a-o. The structural assignments of the compounds 4 a-o were based on the characterization of their ${ }^{1} \mathrm{H} \mathrm{NMR},{ }^{13} \mathrm{C} N M R$, and mass spectra. Satisfactory elemental analyses were obtained (Scheme 1). 
<smiles>CCOC(=O)CC(C)=O</smiles>

(A)

(B)<smiles>CC(=O)c1cc2ccccc2oc1=O</smiles>

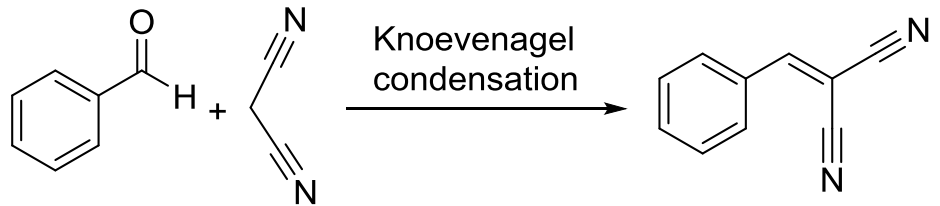

(C)

(D)

(E)<smiles>N#CCC(=O)NN</smiles>
1

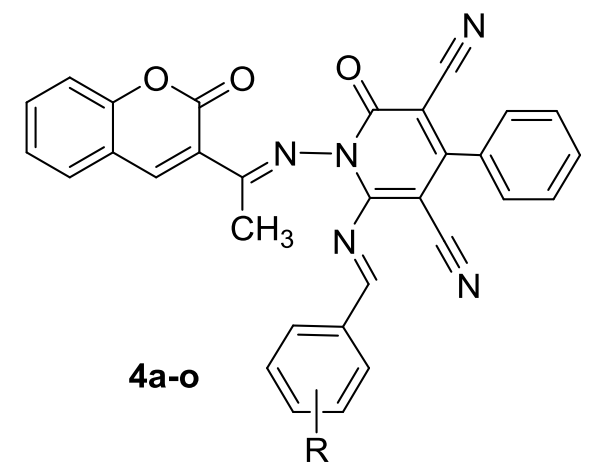

Where $\mathbf{R}=$

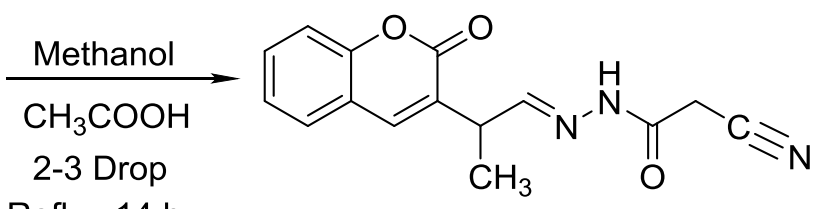

Reflux $14 \mathrm{~h}$

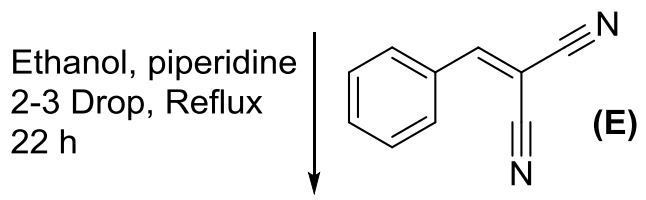<smiles>[R]c1ccc(COc2ccc3oc(=O)c(/C(C)=N/n4c(N)c(C#N)c(-c5ccccc5)c(C#N)c4=O)cc3c2)cc1</smiles>

$\begin{array}{llllll} & \mathbf{R} & & \mathbf{R} & & \mathbf{R} \\ \mathbf{4 a} & -\mathrm{H} & \mathbf{4 f} & -3-\mathrm{NO}_{2} & \mathbf{4 k} & -4-\mathrm{F} \\ \mathbf{4 b} & -2-\mathrm{OH} & \mathbf{4 g} & -4-\mathrm{NO}_{2} & \mathbf{4} & -3-\mathrm{OCH}_{3} \\ \mathbf{4 c} & -3-\mathrm{OH} & \mathbf{4 h} & -2-\mathrm{Cl} & \mathbf{4 m} & -4-\mathrm{OCH}_{3} \\ \mathbf{4 d} & -4-\mathrm{OH} & \mathbf{4 i} & -3-\mathrm{Cl} & \mathbf{4 n} & -3,4,5-\left(\mathrm{OCH}_{3}\right)_{3} \\ \mathbf{4 e} & -2-\mathrm{NO}_{2} & \mathbf{4 j} & -4-\mathrm{Cl} & \mathbf{4 0} & -4-\mathrm{Br}\end{array}$

Scheme 1. Synthetic route for preparation of title compounds (4a-o)

A plausible mechanistic pathway for the formation of compounds 4a-o is suggested in Scheme 2. In first step, hydrazone (A) underwent Michael addition with Knoevenagel product (B) and produced the intermediate $(\mathbf{C})$, which further undergoes intramolecular nucleophilic attack on cyanide carbon followed by annulation to yield intermediate (D). The intermediate (D) transformed to compound (E) by intramolecular electron transfer to nitrogen atom. In the last step, intermediate (E) was transformed to targeted compounds by intermolecular nucleophilic attack on carbonyl carbon of different aromatic aldehydes (Scheme 2). 


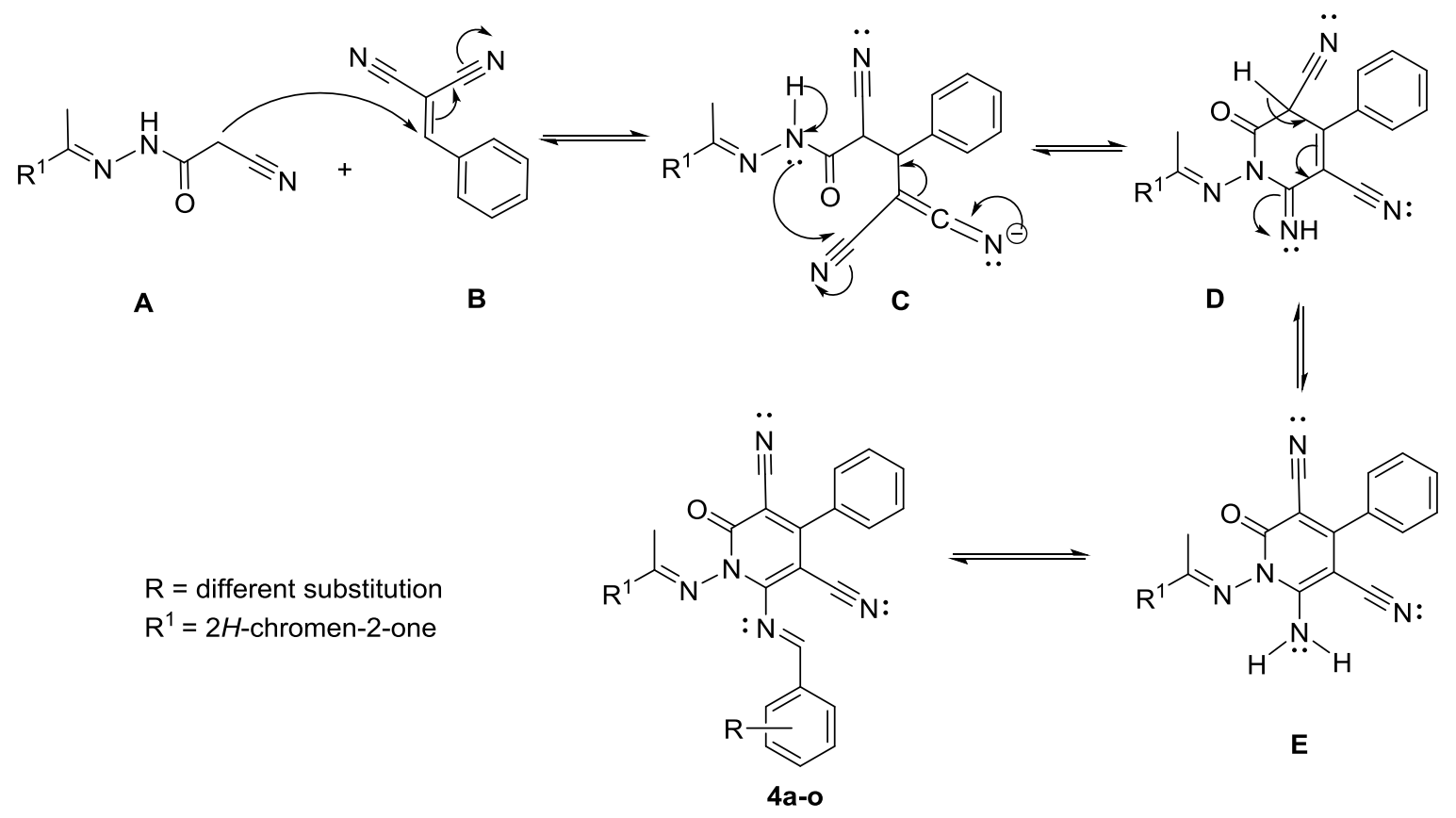

Scheme 2. Plausible mechanistic pathway

\subsection{Characterization of compounds 4a-o}

The structure of compound 4a-o was confirmed on the basis of spectral data. The IR spectrum of compound 4a-o showed strong absorption band at $1700-1730 \mathrm{~cm}^{-1}$ due to $>C=O$ stretching, coumarin ring. Absorption band appeared at 2203-2221 $\mathrm{cm}^{-1}$ due to stretching vibrations corresponding to cyano group and absorption band at 1591-1599, 1551-1562 $\mathrm{cm}^{-1}$ corresponding to $\mathrm{C}=\mathrm{C}, \mathrm{C}=\mathrm{N}$ stretching, aromatic ring. Moreover absorption bands appeared in compounds $4 \mathbf{a}-\mathbf{0}$ at 1251-1268 $\mathrm{cm}^{-1}$ indicated the C-O-C linkage present in coumarin ring. In ${ }^{1} \mathrm{H}$ NMR spectra, the appearance of singlet peaks in compounds 4a-o at $\delta=8.65-9.30$ and 9.05-9.25 ppm was due to one proton of coumarin ring $(\mathrm{C} 4-\mathrm{H})$ and $\mathrm{Ar}-\mathrm{C} \underline{\mathrm{H}}=\mathrm{N}$ - linkage. Three proton of $\mathrm{Ar}-\mathrm{C}\left(\mathrm{CH}_{3}\right)=\mathrm{N}$ - displayed singlet at $\delta=2.03-2.10 \mathrm{ppm}$. Remaining all aromatic protons appeared multiplet in the region $\delta=$ 6.90-8.53 ppm. The ${ }^{13} \mathrm{C}$ NMR spectrum of compound 4a-o showed characteristic signal at $\delta=$ 159.2-159.9 ppm due to carbonyl carbon at coumarine motif as well as the appearance of signal around $\delta=115.5$ (2) ppm was assignable to cyano group of the 2-pyridone ring. Carbon of $-\mathrm{CH}=\mathrm{N}-$ linkage showed signal around $\delta=163.2 \mathrm{ppm}$. The mass spectrum revealed a molecular ion peak in compound 4a-o at $\mathrm{m} / \mathrm{z}=509.15-599.18$ in mass spectra, molecular ion peak was in agreement with proposed molecular weight and elemental analysis.

\subsection{Antimicrobial Results}

The activity of compounds was determined as per the National Committee for Clinical Laboratory Standards (NCCLS) protocol using Mueller-Hinton Broth (Becton-Dickinson, USA) [27,28,29,30]. All the newly synthesized compounds were evaluated against Gram-positive bacteria (Staphylococcus aureus, Staphylococcus pyogenes), Gram-negative bacteria (Escherichia coli, Pseudomonas aeruginosa) and fungi (Candida albicans, Aspergillus niger and Aspergillus clavatus) strains. 
Table 1. Antibacterial and Antifungal activity of compounds 4a-o

\begin{tabular}{|c|c|c|c|c|c|c|c|c|}
\hline \multirow[b]{2}{*}{$\begin{array}{l}\text { Sr. } \\
\text { No. }\end{array}$} & \multirow[b]{2}{*}{$-\mathbf{R}$} & \multicolumn{4}{|c|}{$\begin{array}{c}\text { Minimum inhibitory concentrations for } \\
\text { bacteria } \\
\left(\mathrm{MIC}_{\mathrm{B}}\right) \text { in } \mu \mathrm{g} / \mathrm{ml}\end{array}$} & \multicolumn{3}{|c|}{$\begin{array}{l}\text { Minimum inhibitory } \\
\text { concentration for fungi } \\
\left(\mathrm{MIC}_{\mathrm{F}}\right) \text { in } \mu \mathrm{g} / \mathrm{ml}\end{array}$} \\
\hline & & $\begin{array}{c}\text { E. coli } \\
\text { MTCC } \\
443\end{array}$ & $\begin{array}{l}P . \\
\text { aeruginosa } \\
\text { MTCC } \\
1688\end{array}$ & $\begin{array}{l}\text { S. } \\
\text { aureus } \\
\text { MTCC } \\
96\end{array}$ & $\begin{array}{c}S . \\
\text { pyogenes } \\
\text { MTCC } \\
442\end{array}$ & $\begin{array}{l}\text { C. } \\
\text { albicans } \\
\text { MTCC } \\
227\end{array}$ & $\begin{array}{l}\text { A. niger } \\
\text { MTCC } \\
282\end{array}$ & $\begin{array}{c}\text { A. } \\
\text { clavatus } \\
\text { MTCC } \\
1323\end{array}$ \\
\hline $4 a$ & $-\mathrm{H}$ & $>1000$ & 500 & 1000 & 500 & 500 & 1000 & 250 \\
\hline $4 b$ & $-2-\mathrm{OH}$ & 1000 & 1000 & 500 & 1000 & 500 & 100 & 100 \\
\hline $4 c$ & $-3-\mathrm{OH}$ & 500 & 500 & 250 & $>1000$ & 100 & 50 & 50 \\
\hline $4 d$ & $-4-\mathrm{OH}$ & 250 & 1000 & 500 & 250 & 25 & 12.5 & 50 \\
\hline $4 e$ & $-2-\mathrm{NO}_{2}$ & 100 & 250 & 500 & 100 & $>1000$ & 250 & 500 \\
\hline $4 f$ & $-3-\mathrm{NO}_{2}$ & 50 & 250 & 100 & 50 & 1000 & $>1000$ & $>1000$ \\
\hline $4 \mathrm{~g}$ & $-4-\mathrm{NO}_{2}$ & 12.5 & 25 & 25 & 100 & 1000 & $>1000$ & $>1000$ \\
\hline $4 h$ & $-2-\mathrm{Cl}^{2}$ & 250 & $>1000$ & 250 & 250 & 1000 & 500 & 500 \\
\hline $4 \mathbf{i}$ & $-3-\mathrm{Cl}$ & 100 & 500 & 50 & 1000 & $>1000$ & 500 & 250 \\
\hline $4 \mathbf{j}$ & $-4-\mathrm{Cl}$ & 25 & 12.5 & 100 & 12.5 & $>1000$ & 1000 & 250 \\
\hline $4 k$ & $-4-F$ & 12.5 & 12.5 & 100 & 12.5 & 500 & 500 & $>1000$ \\
\hline 41 & $-3-\mathrm{OCH}_{3}$ & $>1000$ & 500 & 500 & 1000 & 100 & 50 & 250 \\
\hline $4 m$ & $-4-\mathrm{OCH}_{3}$ & 500 & 1000 & 500 & 250 & 50 & 12.5 & 12.5 \\
\hline $4 n$ & $-3,4,5-\left(\mathrm{OCH}_{3}\right)_{3}$ & 1000 & $>1000$ & 250 & 500 & 100 & 25 & 50 \\
\hline 40 & $-4-\mathrm{Br}$ & 50 & 12.5 & 12.5 & 50 & $>1000$ & 500 & 500 \\
\hline & Ampicillin & 100 & 100 & 250 & 100 & - & - & - \\
\hline & Griseofulvin & - & - & - & - & 500 & 100 & 100 \\
\hline
\end{tabular}

\subsubsection{Antibacterial activity}

In preliminary screening, compounds $\mathbf{4 a - 0}$ against the test microbes are listed in Table 1 along with MIC values of reference compounds ampicillin (for bacteria) and griseofulvin (for fungi). Although all coumarine base 2-pyridone derivatives 4a-o were found to show antimicrobial activity against different strains in these two assays, compounds $\mathbf{4 g}, \mathbf{4 j}, \mathbf{4 k}$ and $\mathbf{4 0}$ are clearly outstanding in their antibacterial properties. For several pathogens, these compounds were more active than the reference drugs.

\subsubsection{Antifungal activity}

Compounds 4a-o were tested for antifungal activity in six sets against $C$. albicans, A. niger and A. clavatus at various concentrations of $1000,500,200$ and $100 \mu \mathrm{g} / \mathrm{ml}$ as shown in Table 1 . Synthesized compounds are diluted at $1000 \mu \mathrm{g} \mathrm{ml}{ }^{-1}$ concentration, as a stock solution. The data of antimicrobial evaluation of compounds 4a-o were collected in Table 1. Compound 4d selectively inhibits the growth of $C$. albicans and A. niger. Compound $\mathbf{4 m}$, expressed excellent activity against $A$. niger and $A$. clavatus while compound $4 \mathbf{n}$ exhibited excellent activity against $A$.niger with a two to four fold higher $(12.5-25 \mu \mathrm{g} / \mathrm{ml}) \mathrm{MIC}$ value than the reference drug griseofulvin. Detailed activity results are summarized in Table 1.

\section{Experimental}

\subsection{Materials and methods}

All reactions except those in aqueous media were carried out by standard techniques for the exclusion of moisture. Melting points were determined on an electro thermal melting point apparatus and were reported uncorrected. TLC on silica gel plates (Merck, $60 \mathrm{~F}_{254}$ ) was used for purity checking and reaction monitoring. Elemental analysis $(\% \mathrm{C}, \mathrm{H}, \mathrm{N})$ was carried out by a Perkin-Elmer $2400 \mathrm{CHN}$ analyzer. IR spectra of all compounds were recorded on a Perkin-Elmer FT-IR spectrophotometer in KBr. ${ }^{1} \mathrm{H}$ NMR spectra were recorded on Varian Gemini $300 \mathrm{MHz}$ and ${ }^{13} \mathrm{C}$ NMR spectra are recorded with a Varian Mercury-400 (100 MHz) NMR spectrometer, using tetramethylsilane as the internal reference, with dimethyl sulfoxide (DMSO- $d_{6}$ ) as solvent. Mass spectra were scanned on a Shimadzu LCMS 2010 spectrometer. 


\section{Preparation of 2-cyano- $N^{\prime}-(2-(2-0 x 0-2 H$-chromen-3-yl)propylidene) acetohydrazide (2)}

A mixture of 3-acetyl-2H-chromen-2-one (1) $(0.01 \mathrm{~mol})$ and 2-cyanoacetohydrazide $(0.01 \mathrm{~mol})$ in methanol $(30 \mathrm{~mL})$ was refluxed for $14 \mathrm{~h}$ and then cooled down to room temperature. The separated crystals were filtered, air dried and recrystallized from ethanol $(95 \%)$. Yield: $72 \%$; m.p.: $159{ }^{\circ} \mathrm{C}$; Anal. calcd. for $\mathrm{C}_{15} \mathrm{H}_{13} \mathrm{~N}_{3} \mathrm{O}_{3}$ : C-63.60, H-4.63, N-14.83; Found: C-63.63, H-4.56, N-14.76\%.

Preparation of 6-amino-2-oxo-1-((1-(2-oxo-2H-chromen-3-yl)ethylidene)amino)-4-phenyl-1,2dihydropyridine-3,5-dicarbonitrile (3)

A mixture containing 2-cyano- $N$ '-(2-(2-oxo-2H-chromen-3-yl)propylidene) acetohydrazide (2) $(0.01 \mathrm{~mol}), 2$-benzylidenemalononitrile $(0.01 \mathrm{~mol})$ and 2 drops of piperidine in ethanol $(95 \%)(30$ $\mathrm{mL}$ ) was refluxed for $22 \mathrm{~h}$. The mixture was then cooled down to room temperature and diluted with few drops of water. The crystals formed were filtered, air dried and recrystallized from ethanol. Yield: $68 \%$; m.p.: $190{ }^{\circ} \mathrm{C}$; Anal. Calcd. for $\mathrm{C}_{24} \mathrm{H}_{15} \mathrm{~N}_{5} \mathrm{O}_{3}$ : C-68.40, H-3.59, N-16.62; Found: C-68.27, H-3.64, N-16.69\%.

General preparation of 6-((benzylidene)amino)-2-oxo-1-((1-(2-oxo-2H-chromen-3-yl) ethylidene) amino)-4-phenyl-1,2-dihydropyridine-3,5-dicarbonitrile (4a-o)

Compound (3) $(0.01 \mathrm{~mol})$, benzaldehyde $(0.01 \mathrm{~mol})$ and ethanol $(95 \%)(30 \mathrm{~mL})$ were taken in a round bottom flask and refluxed for $12 \mathrm{~h}$. Separated solid was filtered, dried and recrystallized from ethanol.

\section{6-((benzylidene)amino)-2-oxo-1-((1-(2-0xo-2H-chromen-3-yl)ethylidene)amino)-4-phenyl-1,2- dihydropyridine-3,5-dicarbonitrile (4a)}

Yield: 69 \%, m.p. $223-227{ }^{\circ} \mathrm{C}$. IR (KBr, cm ${ }^{-1}$ ): 3133, 3029 (C-H stretching, aromatic ring), 2917 ( $\left.\mathrm{C}-\mathrm{H}, \mathrm{CH}_{3}\right), 2205$ ( $-\mathrm{C} \equiv \mathrm{N}$ stretching, nitrile group), 1727 ( $>\mathrm{C}=\mathrm{O}$ stretching, coumarin ring), 1559, $1595(>\mathrm{C}=\mathrm{N}-,>\mathrm{C}=\mathrm{C}<$ stretching, aromatic ring $), 1586,1402(\mathrm{C}-\mathrm{H}$ bending, $-\mathrm{CH}=\mathrm{N}$ linkage $), 1251$ (C-O-C coumarin ring). ${ }^{1} \mathrm{H}$ NMR $\left(300 \mathrm{MHz}, \mathrm{DMSO}-d_{6}, \delta \mathrm{ppm}\right): 9.10(\mathrm{~s}, 1 \mathrm{H}, \mathrm{Ar}-\mathrm{CH}=\mathrm{N}-), 8.99$ (s, $1 \mathrm{H}, \mathrm{C} 4$ proton of coumarin), 7.13-7.83 $(\mathrm{m}, 14 \mathrm{H}$, Ar-H of coumarin ring and phenyl ring), 2.03 (s, $\left.3 \mathrm{H},-\mathrm{C}\left(\mathrm{C}_{3}\right)=\mathrm{N}-\right) .{ }^{13} \mathrm{C}$ NMR $\left(100 \mathrm{MHz}, \mathrm{DMSO}-d_{6}, \delta \mathrm{ppm}\right): 169.1,163.5,160.2,159.9,155.3$, $153.6,153.2$, 133.3, 133.1, 132.8, 131.1, 129.4, 129.4, 128.7, 128.7, 128.5, 128.5, 128.1, 128.1, 128.0, 127.6, 127.6, 125.7, 123.5, 118.3, 116.2, 115.6, 115.6, 115.2, 114.9, 4.6. MS: m/z 509.15 $\left(\mathrm{M}^{+}\right.$). Anal. calcd. For $\mathrm{C}_{31} \mathrm{H}_{19} \mathrm{~N}_{5} \mathrm{O}_{3}, \mathrm{C}, 73.08 ; \mathrm{H}, 3.76 ; \mathrm{N}, 13.75 \%$. Found: $\mathrm{C}, 73.02 ; \mathrm{H}, 3.79$; N, $13.71 \%$.

\section{6-((2-hydroxybenzylidene)amino)-2-oxo-1-((1-(2-oxo-2H-chromen-3-yl)ethylidene)amino)- 4-phenyl-1,2-dihydropyridine-3,5-dicarbonitrile (4b)}

Yield: 77 \%, m.p. $174-177{ }^{\circ} \mathrm{C}$. IR (KBr, cm $\left.{ }^{-1}\right)$ : 3415 (O-H, Ar-OH), 3135, 3034 (C-H stretching, aromatic ring), $2914\left(\mathrm{C}-\mathrm{H}, \mathrm{CH}_{3}\right), 2209(-\mathrm{C} \equiv \mathrm{N}$ stretching, nitrile group), $1708(>\mathrm{C}=\mathrm{O}$ stretching, coumarin ring), 1551, $1599(>\mathrm{C}=\mathrm{N}-,>\mathrm{C}=\mathrm{C}<$ stretching, aromatic ring), $1404(\mathrm{C}-\mathrm{H}$ bending, $-\mathrm{CH}=\mathrm{N}$ linkage), 1252 (C-O-C coumarin ring). ${ }^{1} \mathrm{H}$ NMR (300 MHz, DMSO- $d_{6}, \delta$ ppm): 11.02 (s, 1H, Ar$\mathrm{OH}), 9.18(\mathrm{~s}, 1 \mathrm{H}, \mathrm{Ar}-\mathrm{CH}=\mathrm{N}-), 9.25(\mathrm{~s}, 1 \mathrm{H}, \mathrm{C} 4$ proton of coumarin $), 6.90-7.81(\mathrm{~m}, 13 \mathrm{H}, \mathrm{Ar}-\mathrm{H}$ of coumarin ring and phenyl ring), 2.05 (s, $\left.3 \mathrm{H}, \mathrm{Ar}-\mathrm{C}\left(\mathrm{CH}_{3}\right)=\mathrm{N}-\right) .{ }^{13} \mathrm{C}$ NMR $\left(100 \mathrm{MHz}, \mathrm{DMSO}-d_{6}, \delta\right.$ ppm): 169.6, 163.3, 161.5, 160.4, 159.7, 155.5, 153.3, 153.1, 133.7, 132.6, 132.4, 132.6, 128.7, 128.7, 128.4, 128.4, 128.1, 127.7, 127.7, 125.6, 123.6, 121.6, 118.6, 118.2, 117.5, 116.3, 115.6, 115.6, 115.5, 114.5, 4.6. MS: m/z 525.14 $\left(\mathrm{M}^{+}\right)$. Anal. calcd. For $\mathrm{C}_{31} \mathrm{H}_{19} \mathrm{~N}_{5} \mathrm{O}_{4}, \mathrm{C}, 70.85 ; \mathrm{H}, 3.64 ; \mathrm{N}$, $13.33 \%$. Found: C, 70.82; H, 3.69; N, $13.36 \%$.

\section{6-((3-hydroxybenzylidene)amino)-2-oxo-1-((1-(2-oxo-2H-chromen-3-yl)ethylidene)amino)- 4-phenyl-1,2-dihydropyridine-3,5-dicarbonitrile (4c)}

Yield: $71 \%$, m.p. $198-202{ }^{\circ} \mathrm{C}$. IR $\left(\mathrm{KBr}, \mathrm{cm}^{-1}\right)$ : 3405 (O-H, Ar-OH), 3131, 3035 (C-H stretching, aromatic ring), $2914\left(\mathrm{C}-\mathrm{H}, \mathrm{CH}_{3}\right), 2212(-\mathrm{C} \equiv \mathrm{N}$ stretching, nitrile group), $1713(>\mathrm{C}=\mathrm{O}$ stretching, coumarin ring), 1562, $1599(>\mathrm{C}=\mathrm{N}-,>\mathrm{C}=\mathrm{C}<$ stretching, aromatic ring), $1406(\mathrm{C}-\mathrm{H}$ bending, $-\mathrm{CH}=\mathrm{N}$ linkage), 1266 (C-O-C coumarin ring). ${ }^{1} \mathrm{H}$ NMR (300 MHz, DMSO- $d_{6}, \delta$ ppm): 9.49 (s, 1H, Ar- 
$\mathrm{OH}), 9.22(\mathrm{~s}, 1 \mathrm{H}, \mathrm{Ar}-\mathrm{CH}=\mathrm{N}-), 8.90(\mathrm{~s}, 1 \mathrm{H}, \mathrm{C} 4$ proton of coumarin), 6.94-7.84 (m, 13H, Ar-H of coumarin ring and phenyl ring), $2.05\left(\mathrm{~s}, 3 \mathrm{H}, \mathrm{Ar}-\mathrm{C}\left(\mathrm{CH}_{3}\right)=\mathrm{N}-\right) .{ }^{13} \mathrm{C}$ NMR $\left(100 \mathrm{MHz}, \mathrm{DMSO}-d_{6}, \delta\right.$ ppm): 169.2, 163.9, 160.5, 159.7, 158.7, 155.7, 153.7, 153.3, 135.3, 133.6, 132.2, 130.6, 128.5, $128.5,128.2,128.2,128.0,127.8,127.8,125.1,123.6,121.5,118.4,118.2,116.3,115.7,115.7$, 115.3, 114.6, 114.5, 4.6. MS: m/z 525.14 (M $\mathrm{M}^{+}$). Anal. calcd. For $\mathrm{C}_{31} \mathrm{H}_{19} \mathrm{~N}_{5} \mathrm{O}_{4}, \mathrm{C}, 70.85 ; \mathrm{H}, 3.64 ; \mathrm{N}$, $13.33 \%$. Found: $\mathrm{C}, 70.87 ; \mathrm{H}, 3.61 ; \mathrm{N}, 13.35 \%$.

\section{6-((4-hydroxybenzylidene)amino)-2-oxo-1-((1-(2-oxo-2H-chromen-3-yl)ethylidene)amino)- 4-phenyl-1,2-dihydropyridine-3,5-dicarbonitrile (4d)}

Yield: 63 \%, m.p. $162-166^{\circ} \mathrm{C}$. IR $\left(\mathrm{KBr}, \mathrm{cm}^{-1}\right)$ : 3419 (O-H, Ar-OH), 3127, 3039 (C-H stretching, aromatic ring), $2918\left(\mathrm{C}-\mathrm{H}, \mathrm{CH}_{3}\right), 2206(-\mathrm{C} \equiv \mathrm{N}$ stretching, nitrile group), $1704(>\mathrm{C}=\mathrm{O}$ stretching, coumarin ring), 1551, $1596(>\mathrm{C}=\mathrm{N}-,>\mathrm{C}=\mathrm{C}<$ stretching, aromatic ring), $1409(\mathrm{C}-\mathrm{H}$ bending, $-\mathrm{CH}=\mathrm{N}$ linkage), 1261 (C-O-C coumarin ring). ${ }^{1} \mathrm{H}$ NMR $\left(300 \mathrm{MHz}, \mathrm{DMSO}-d_{6}, \delta \mathrm{ppm}\right): 9.74(\mathrm{~s}, 1 \mathrm{H}, \mathrm{Ar}-$ $\mathrm{OH}), 9.20(\mathrm{~s}, 1 \mathrm{H}, \mathrm{Ar}-\mathrm{CH}=\mathrm{N}-), 8.65(\mathrm{~s}, 1 \mathrm{H}, \mathrm{C} 4$ proton of coumarin), 6.91-7.89 (m, 13H, Ar-H of coumarin ring and phenyl ring), $2.03\left(\mathrm{~s}, 3 \mathrm{H}, \mathrm{Ar}-\mathrm{C}\left(\mathrm{CH}_{3}\right)=\mathrm{N}-\right) .{ }^{13} \mathrm{C}$ NMR $\left(100 \mathrm{MHz}, \mathrm{DMSO}-d_{6}, \delta\right.$ ppm): 169.5, 163.5, 160.9, 160.7, 159.7, 155.3, 153.5, 153.0, 133.7, 132.8, 130.4, 130.4, 128.8, $128.8,128.5,128.5,128.1,127.7,127.7,126.5,125.4,123.6,118.4,116.2,116.0,116.0,115.6$, 115.6, 115.1, 114.3, 4.4. MS: m/z 525.14 (M $\mathrm{M}^{+}$). Anal. calcd. For $\mathrm{C}_{31} \mathrm{H}_{19} \mathrm{~N}_{5} \mathrm{O}_{4}, \mathrm{C}, 70.85 ; \mathrm{H}, 3.64 ; \mathrm{N}$, $13.33 \%$. Found: C, 70.78; H, 3.59; N, 13.26\%.

\section{6-((2-nitrobenzylidene)amino)-2-oxo-1-((1-(2-oxo-2H-chromen-3-yl)ethylidene)amino)- 4-phenyl-1,2-dihydropyridine-3,5-dicarbonitrile (4e)}

Yield: $68 \%$, m.p. $236-239{ }^{\circ} \mathrm{C}$. IR (KBr, cm $\left.{ }^{-1}\right)$ : 3136, 3031 (C-H stretching, aromatic ring), 2920 (C-H, $\left.\mathrm{CH}_{3}\right), 2205$ ( $\mathrm{C} \equiv \mathrm{N}$ stretching, nitrile group), 1711 ( $>\mathrm{C}=\mathrm{O}$ stretching, coumarin ring), 1553, $1593(>\mathrm{C}=\mathrm{N}-,>\mathrm{C}=\mathrm{C}<$ stretching, aromatic ring $), 1484,1356\left(-\mathrm{NO}_{2}\right), 1401(\mathrm{C}-\mathrm{H}$ bending, $-\mathrm{CH}=\mathrm{N}$ linkage), 1262 (C-O-C coumarin ring). ${ }^{1} \mathrm{H}$ NMR (300 MHz, DMSO- $d_{6}, \delta$ ppm): 9.07 (s, 1H, Ar$\mathrm{CH}=\mathrm{N}-), 9.30(\mathrm{~s}, 1 \mathrm{H}, \mathrm{C} 4$ proton of coumarin), 7.15-8.09 $(\mathrm{m}, 13 \mathrm{H}, \mathrm{Ar}-\mathrm{H}$ of coumarin ring and phenyl ring), $2.05\left(\mathrm{~s}, 3 \mathrm{H},-\mathrm{C}\left(\mathrm{C}_{3}\right)=\mathrm{N}-\right) .{ }^{13} \mathrm{C}$ NMR $\left(100 \mathrm{MHz}, \mathrm{DMSO}-d_{6}, \delta \mathrm{ppm}\right): 169.5,163.9$, $160.3,159.5,155.4,153.5,153.1,147.4,134.7,133.6,132.3,131.4,130.3,128.6,128.6,128.3$, 128.3, 128.2, 128.0, 127.5, 127.5, 125.3, 124.1, 123.4, 118.5, 116.3, 115.5, 115.5, 115.1, 114.6, 4.7. MS: $\mathrm{m} / \mathrm{z} 554.13\left(\mathrm{M}^{+}\right)$. Anal. calcd. For $\mathrm{C}_{31} \mathrm{H}_{18} \mathrm{~N}_{6} \mathrm{O}_{5}, \mathrm{C}, 67.15 ; \mathrm{H}, 3.27 ; \mathrm{N}, 15.16 \%$. Found: $\mathrm{C}$, $67.21 ; \mathrm{H}, 3.18 ; \mathrm{N}, 15.23 \%$.

\section{6-((3-nitrobenzylidene)amino)-2-oxo-1-((1-(2-oxo-2H-chromen-3-yl)ethylidene)amino)- 4-phenyl-1,2-dihydropyridine-3,5-dicarbonitrile (4f)}

Yield: $61 \%$, m.p. $244-247{ }^{\circ} \mathrm{C}$. IR (KBr, cm $\left.{ }^{-1}\right)$ : 3130, 3034 (C-H stretching, aromatic ring), 2924 (C-H, $\left.\mathrm{CH}_{3}\right), 2221(-\mathrm{C} \equiv \mathrm{N}$ stretching, nitrile group), 1700 ( $>\mathrm{C}=\mathrm{O}$ stretching, coumarin ring), 1553, $1598(>\mathrm{C}=\mathrm{N}-,>\mathrm{C}=\mathrm{C}<$ stretching, aromatic ring $), 1480,1352\left(-\mathrm{NO}_{2}\right), 1403(\mathrm{C}-\mathrm{H}$ bending, $-\mathrm{CH}=\mathrm{N}$ linkage), 1266 (C-O-C coumarin ring). ${ }^{1} \mathrm{H}$ NMR (300 MHz, DMSO- $d_{6}, \delta$ ppm): 9.15 (s, $1 \mathrm{H}, \mathrm{Ar}-$ $\mathrm{CH}=\mathrm{N}-), 8.87(\mathrm{~s}, 1 \mathrm{H}, \mathrm{C} 4$ proton of coumarin), 7.19-8.53 (m, 13H, Ar-H of coumarin ring and phenyl ring), $2.08\left(\mathrm{~s}, 3 \mathrm{H},-\mathrm{C}\left(\mathrm{CH}_{3}\right)=\mathrm{N}-\right) .{ }^{13} \mathrm{C}$ NMR (100 MHz, DMSO-d 6 , $\delta$ ppm): 169.7, 163.3, 160.1 , 159.2, 155.9, 153.4, 148.1, 135.4, 134.9, 133.7, 132.7, 129.4, 128.9, 128.9, 128.7, 128.7, 128.5, 128.0, 127.7, 127.7, 126.3, 125.4, 123.6, 121.5, 118.4, 116.3, 115.7, 115.7, 115.1, 114.9, 4.7. MS: $\mathrm{m} / \mathrm{z} 554.13\left(\mathrm{M}^{+}\right)$. Anal. calcd. For $\mathrm{C}_{31} \mathrm{H}_{18} \mathrm{~N}_{6} \mathrm{O}_{5}, \mathrm{C}, 67.15 ; \mathrm{H}, 3.27 ; \mathrm{N}, 15.16 \%$. Found: $\mathrm{C}$, $67.12 ; \mathrm{H}, 3.21 ; \mathrm{N}, 15.21 \%$.

\section{6-((4-nitrobenzylidene)amino)-2-oxo-1-((1-(2-oxo-2H-chromen-3-yl)ethylidene)amino)- 4-phenyl-1,2-dihydropyridine-3,5-dicarbonitrile (4g)}

Yield: $72 \%$, m.p. $221-225{ }^{\circ} \mathrm{C}$. IR (KBr, cm $\left.{ }^{-1}\right)$ : 3137, 3031 (C-H stretching, aromatic ring), 2926 (C-H, $\left.\mathrm{CH}_{3}\right), 2215$ ( $\mathrm{C} \equiv \mathrm{N}$ stretching, nitrile group), 1730 ( $>\mathrm{C}=\mathrm{O}$ stretching, coumarin ring), 1557, $1594\left(>\mathrm{C}=\mathrm{N}-,>\mathrm{C}=\mathrm{C}<\right.$ stretching, aromatic ring), 1487, $1359\left(-\mathrm{NO}_{2}\right), 1268(\mathrm{C}-\mathrm{O}-\mathrm{C}$ coumarin ring), 1405 (C-H bending, -CH=N linkage). ${ }^{1} \mathrm{H}$ NMR (300 MHz, DMSO- $d_{6}, \delta$ ppm): 9.12 (s, $1 \mathrm{H}, \mathrm{Ar}-$ $\mathrm{CH}=\mathrm{N}-), 8.70(\mathrm{~s}, 1 \mathrm{H}, \mathrm{C} 4$ proton of coumarin), 7.22-8.37 (m, 13H, Ar-H of coumarin ring and 
phenyl ring), $2.03\left(\mathrm{~s}, 3 \mathrm{H},-\mathrm{C}\left(\mathrm{CH}_{3}\right)=\mathrm{N}-\right) .{ }^{13} \mathrm{C}$ NMR (100 MHz, DMSO- $\left.d_{6}, \delta \mathrm{ppm}\right): 169.1,163.6$, $160.3,159.4,155.7,153.5,153.1,150.4,139.9,133.2,132.4,128.7,128.7,128.5,128.5,128.3$, 127.5, 127.5, 127.3, 127.3, 125.8, 124.2, 124.2, 123.5, 118.3, 116.2, 115.9, 115.9, 115.5, 114.4, 4.6. MS: $\mathrm{m} / \mathrm{z} 554.13\left(\mathrm{M}^{+}\right)$. Anal. calcd. For $\mathrm{C}_{31} \mathrm{H}_{18} \mathrm{~N}_{6} \mathrm{O}_{5}, \mathrm{C}, 67.15 ; \mathrm{H}, 3.27 ; \mathrm{N}, 15.16 \%$. Found: C, $67.07 ; \mathrm{H}, 3.34 ; \mathrm{N}, 15.11 \%$.

\section{6-((2-chlorobenzylidene)amino)-2-oxo-1-((1-(2-oxo-2H-chromen-3-yl)ethylidene)amino)- 4-phenyl-1,2-dihydropyridine-3,5-dicarbonitrile (4h)}

Yield: $73 \%$, m.p. $217-221{ }^{\circ} \mathrm{C}$. IR (KBr, cm $\left.{ }^{-1}\right)$ : 3139, 3029 (C-H stretching, aromatic ring), 2928 (C-H, $\left.\mathrm{CH}_{3}\right), 2208(-\mathrm{C} \equiv \mathrm{N}$ stretching, nitrile group), 1706 ( $>\mathrm{C}=\mathrm{O}$ stretching, coumarin ring), 1560, $1594(>\mathrm{C}=\mathrm{N}-,>\mathrm{C}=\mathrm{C}<$ stretching, aromatic ring), 1267 (C-O-C coumarin ring), 1408 (C-H bending, $-\mathrm{CH}=\mathrm{N}$ linkage), $754(\mathrm{C}-\mathrm{Cl}) .{ }^{1} \mathrm{H}$ NMR $\left(300 \mathrm{MHz}, \mathrm{DMSO}-d_{6}, \delta \mathrm{ppm}\right): 9.18(\mathrm{~s}, 1 \mathrm{H}, \mathrm{Ar}-\mathrm{CH}=\mathrm{N}-)$, 8.94 (s, 1H, C4 proton of coumarin), 7.14-7.90 (m, 13H, Ar-H of coumarin ring and phenyl ring), $2.06\left(\mathrm{~s}, 3 \mathrm{H},-\mathrm{C}\left(\mathrm{CH}_{3}\right)=\mathrm{N}-\right) .{ }^{13} \mathrm{C} \mathrm{NMR}\left(100 \mathrm{MHz}, \mathrm{DMSO}-d_{6}, \delta \mathrm{ppm}\right): 169.9,163.5,160.3,159.6$, $155.3,153.4,153.2,135.3,134.5,133.7,132.3,131.4,130.6,128.8,128.8,128.6,128.6,128.1$, $127.7,127.7,127.5,127.4,125.8,123.6,118.3,116.7,115.9,115.9,115.5,114.5,4.6 . \mathrm{MS}: \mathrm{m} / \mathrm{z}$ $543.11\left(\mathrm{M}^{+}\right)$. Anal. calcd. For $\mathrm{C}_{31} \mathrm{H}_{18} \mathrm{ClN}_{5} \mathrm{O}_{3}, \mathrm{C}, 68.45 ; \mathrm{H}, 3.34 ; \mathrm{N}, 12.87$ \%. Found: $\mathrm{C}, 68.51 ; \mathrm{H}$, $3.29 ; \mathrm{N}, 12.96 \%$.

\section{6-((3-chlorobenzylidene)amino)-2-oxo-1-((1-(2-oxo-2H-chromen-3-yl)ethylidene)amino)- 4-phenyl-1,2-dihydropyridine-3,5-dicarbonitrile (4i)}

Yield: $65 \%$, m.p. $226-230{ }^{\circ} \mathrm{C}$. IR (KBr, cm $\left.{ }^{-1}\right)$ : 3143, 3025 (C-H stretching, aromatic ring), 2923 (C-H, $\left.\mathrm{CH}_{3}\right), 2203(-\mathrm{C} \equiv \mathrm{N}$ stretching, nitrile group), 1716 ( $>\mathrm{C}=\mathrm{O}$ stretching, coumarin ring), 1557, $1597(>\mathrm{C}=\mathrm{N}-,>\mathrm{C}=\mathrm{C}<$ stretching, aromatic ring), $1410(\mathrm{C}-\mathrm{H}$ bending, $-\mathrm{CH}=\mathrm{N}$ linkage $), 1258(\mathrm{C}-\mathrm{O}-$ $\mathrm{C}$ coumarin ring), $757(\mathrm{C}-\mathrm{Cl}) .{ }^{1} \mathrm{H}$ NMR $\left(300 \mathrm{MHz}, \mathrm{DMSO}-d_{6}, \delta \mathrm{ppm}\right): 9.10(\mathrm{~s}, 1 \mathrm{H}, \mathrm{Ar}-\mathrm{CH}=\mathrm{N}-)$, $9.11(\mathrm{~s}, 1 \mathrm{H}, \mathrm{C} 4$ proton of coumarin), 7.16-7.89 (m, 13H, Ar-H of coumarin ring and phenyl ring), $2.09\left(\mathrm{~s}, 3 \mathrm{H},-\mathrm{C}\left(\mathrm{C}_{3}\right)=\mathrm{N}-\right) .{ }^{13} \mathrm{C}$ NMR $\left(100 \mathrm{MHz}, \mathrm{DMSO}-d_{6}, \delta \mathrm{ppm}\right): 169.4,163.9,160.5,159.7$, $155.3,153.4,153.2,133.6,133.5,133.1,132.7,132.6,130.3,128.7,128.7,128.5,128.5,128.2$, $127.8,127.8,127.3,126.7,125.6,123.4,118.2,116.3,115.7,115.7,115.3,114.6,4.7 . \mathrm{MS}: \mathrm{m} / \mathrm{z}$ $543.11\left(\mathrm{M}^{+}\right)$. Anal. calcd. For $\mathrm{C}_{31} \mathrm{H}_{18} \mathrm{ClN}_{5} \mathrm{O}_{3}, \mathrm{C}, 68.45 ; \mathrm{H}, 3.34 ; \mathrm{N}, 12.87 \%$. Found: $\mathrm{C}, 68.36$; $\mathrm{H}$, $3.26 ; \mathrm{N}, 12.81 \%$.

\section{6-((4-chlorobenzylidene)amino)-2-oxo-1-((1-(2-oxo-2H-chromen-3-yl)ethylidene)amino)- 4-phenyl-1,2-dihydropyridine-3,5-dicarbonitrile $(4 \mathbf{j})$}

Yield: $62 \%$, m.p. $239-244{ }^{\circ} \mathrm{C}$. IR (KBr, cm $\left.{ }^{-1}\right): 3137,3021$ (C-H stretching, aromatic ring), 2930 (C-H, $\left.\mathrm{CH}_{3}\right), 2217$ ( $\mathrm{C} \equiv \mathrm{N}$ stretching, nitrile group), 1722 ( $>\mathrm{C}=\mathrm{O}$ stretching, coumarin ring), 1559, $1591(>\mathrm{C}=\mathrm{N}-,>\mathrm{C}=\mathrm{C}<$ stretching, aromatic ring), $1416(\mathrm{C}-\mathrm{H}$ bending, $-\mathrm{CH}=\mathrm{N}$ linkage $), 1255(\mathrm{C}-\mathrm{O}-$ $\mathrm{C}$ coumarin ring), $751(\mathrm{C}-\mathrm{Cl}) .{ }^{\mathrm{T}} \mathrm{H}$ NMR $\left(300 \mathrm{MHz}, \mathrm{DMSO}-d_{6}, \delta \mathrm{ppm}\right): 9.20(\mathrm{~s}, 1 \mathrm{H}, \mathrm{Ar}-\mathrm{CH}=\mathrm{N}-)$, $9.15(\mathrm{~s}, 1 \mathrm{H}, \mathrm{C} 4$ proton of coumarin), 7.14-7.87 (m, 13H, Ar-H of coumarin ring and phenyl ring), $2.06\left(\mathrm{~s}, 3 \mathrm{H},-\mathrm{C}\left(\mathrm{CH}_{3}\right)=\mathrm{N}-\right) .{ }^{13} \mathrm{C} \mathrm{NMR}\left(100 \mathrm{MHz}, \mathrm{DMSO}-d_{6}, \delta \mathrm{ppm}\right): 169.7,163.6,160.2,159.6$, $155.5,153.5,153.4,136.6,133.3,132.3,131.7,130.6,130.6,128.7,128.7,128.7,128.7,128.4$, $128.4,128.2,127.7,127.7,125.7,123.5,118.3,116.5,115.9,115.9,115.6,114.4,4.5 . \mathrm{MS}: \mathrm{m} / \mathrm{z}$ $543.11\left(\mathrm{M}^{+}\right)$. Anal. calcd. For $\mathrm{C}_{31} \mathrm{H}_{18} \mathrm{ClN}_{5} \mathrm{O}_{3}, \mathrm{C}, 68.45 ; \mathrm{H}, 3.34 ; \mathrm{N}, 12.87 \%$. Found: $\mathrm{C}, 68.59$; $\mathrm{H}$, $3.42 ; \mathrm{N}, 12.98 \%$.

\section{6-((4-fluorobenzylidene)amino)-2-oxo-1-((1-(2-oxo-2H-chromen-3-yl)ethylidene)amino)- 4-phenyl-1,2-dihydropyridine-3,5-dicarbonitrile (4k)}

Yield: 69 \%, m.p. $243-246{ }^{\circ} \mathrm{C}$. IR $\left(\mathrm{KBr}, \mathrm{cm}^{-1}\right): 3134,3029$ (C-H stretching, aromatic ring), 2934 $\left(\mathrm{C}-\mathrm{H}, \mathrm{CH}_{3}\right), 1135(\mathrm{C}-\mathrm{F}), 2219(-\mathrm{C} \equiv \mathrm{N}$ stretching, nitrile group), 1719 ( $>\mathrm{C}=\mathrm{O}$ stretching, coumarin ring), 1561, $1599(>\mathrm{C}=\mathrm{N}-,>\mathrm{C}=\mathrm{C}<$ stretching, aromatic ring), $1419(\mathrm{C}-\mathrm{H}$ bending, $-\mathrm{CH}=\mathrm{N}$ linkage $)$, 1260 (C-O-C coumarin ring). ${ }^{1} \mathrm{H}$ NMR (300 MHz, DMSO- $\left.d_{6}, \delta \mathrm{ppm}\right): 9.25$ (s, 1H, Ar-CH=N-), $9.07(\mathrm{~s}, 1 \mathrm{H}, \mathrm{C} 4$ proton of coumarin), 7.11-7.92 (m, 13H, Ar-H of coumarin ring and phenyl ring), $2.10\left(\mathrm{~s}, 3 \mathrm{H},-\mathrm{C}\left(\mathrm{C}_{3}\right)=\mathrm{N}-\right) .{ }^{13} \mathrm{C} \mathrm{NMR}\left(100 \mathrm{MHz}, \mathrm{DMSO}-d_{6}, \delta \mathrm{ppm}\right): 169.9,165.6,163.2,160.6$, 
$159.8,155.9,153.7,153.4,133.9,132.1,130.9,130.9,129.6,128.7,128.7,128.5,128.5,128.1$, $127.4,127.4,125.4,123.2,118.5,116.4,115.5,115.5,115.3,115.3,115.1,114.9,4.7 . \mathrm{MS}: \mathrm{m} / \mathrm{z}$ $527.14\left(\mathrm{M}^{+}\right)$. Anal. calcd. For $\mathrm{C}_{31} \mathrm{H}_{18} \mathrm{FN}_{5} \mathrm{O}_{3}, \mathrm{C}, 70.58 ; \mathrm{H}, 3.44 ; \mathrm{N}, 13.28 \%$. Found: $\mathrm{C}, 70.66 ; \mathrm{H}$, $3.52 ; \mathrm{N}, 13.34 \%$.

\section{6-((3-methoxybenzylidene)amino)-2-oxo-1-((1-(2-oxo-2H-chromen-3-yl)ethylidene)amino)- 4-phenyl-1,2-dihydropyridine-3,5-dicarbonitrile (4I)}

Yield: 74 \%, m.p. $206-209{ }^{\circ} \mathrm{C}$. IR $\left(\mathrm{KBr}, \mathrm{cm}^{-1}\right)$ : 3133, 3036 (C-H stretching, aromatic ring), 2939 $\left(\mathrm{C}-\mathrm{H}, \mathrm{CH}_{3}\right), 2214(-\mathrm{C} \equiv \mathrm{N}$ stretching, nitrile group), $1709(>\mathrm{C}=\mathrm{O}$ stretching, coumarin ring), 1558, $1595(>\mathrm{C}=\mathrm{N}-,>\mathrm{C}=\mathrm{C}<$ stretching, aromatic ring), $1426(\mathrm{C}-\mathrm{H}$ bending, $-\mathrm{CH}=\mathrm{N}$ linkage $), 1263$ (C-O$\mathrm{C}$ coumarin ring), 1214, 1152 (C-O-C). ${ }^{1} \mathrm{H}$ NMR $\left(300 \mathrm{MHz}, \mathrm{DMSO}-d_{6}, \delta \mathrm{ppm}\right): 9.05(\mathrm{~s}, 1 \mathrm{H}, \mathrm{Ar}-$ $\mathrm{CH}=\mathrm{N}-), 8.77(\mathrm{~s}, 1 \mathrm{H}, \mathrm{C} 4$ proton of coumarin), $7.03-7.82(\mathrm{~m}, 13 \mathrm{H}, \mathrm{Ar}-\mathrm{H}$ of coumarin ring and phenyl ring), $3.73\left(\mathrm{~s}, 3 \mathrm{H},-\mathrm{OCH}_{3}\right), 2.05\left(\mathrm{~s}, 3 \mathrm{H},-\mathrm{C}\left(\mathrm{CH}_{3}\right)=\mathrm{N}-\right) .{ }^{13} \mathrm{C} \mathrm{NMR}\left(100 \mathrm{MHz}, \mathrm{DMSO}-d_{6}, \delta\right.$ ppm): 169.1, 163.9, 162.6, 160.4, 159.8, 155.9, 153.1, 153.0, 133.9, 132.6, 130.5, 130.5, 128.8, $128.8,128.5,128.5,128.1,127.6,127.6,126.6,125.6,123.9,118.2,116.3,115.5,115.5,115.1$, 114.8, 114.5, 114.5, 55.4, 4.6. MS: $\mathrm{m} / \mathrm{z} 539.16\left(\mathrm{M}^{+}\right)$. Anal. calcd. For $\mathrm{C}_{32} \mathrm{H}_{21} \mathrm{~N}_{5} \mathrm{O}_{4}, \mathrm{C}, 71.24 ; \mathrm{H}$, $3.92 ;$ N, $12.98 \%$. Found: C, 71.29; H, 3.83; N, $12.91 \%$.

\section{6-((4-methoxybenzylidene)amino)-2-oxo-1-((1-(2-oxo-2H-chromen-3-yl)ethylidene)amino)- 4-phenyl-1,2-dihydropyridine-3,5-dicarbonitrile (4m)}

Yield: 70 \%, m.p. $214-218{ }^{\circ} \mathrm{C}$. IR (KBr, cm $\left.{ }^{-1}\right)$ : 3126, 3041 (C-H stretching, aromatic ring), 2943 (C-H, $\left.\mathrm{CH}_{3}\right), 2218$ ( $-\mathrm{C} \equiv \mathrm{N}$ stretching, nitrile group), 1717 ( $>\mathrm{C}=\mathrm{O}$ stretching, coumarin ring), 1555, $1597(>\mathrm{C}=\mathrm{N}-,>\mathrm{C}=\mathrm{C}<$ stretching, aromatic ring), $1429(\mathrm{C}-\mathrm{H}$ bending, $-\mathrm{CH}=\mathrm{N}$ linkage), 1254 (C-O$\mathrm{C}$ coumarin ring), 1210, 1155 (C-O-C). ${ }^{1} \mathrm{H}$ NMR (300 MHz, DMSO- $d_{6}, \delta$ ppm): 9.20 (s, 1H, Ar$\mathrm{CH}=\mathrm{N}-), 9.10(\mathrm{~s}, 1 \mathrm{H}, \mathrm{C} 4$ proton of coumarin), 7.05-7.88 $(\mathrm{m}, 13 \mathrm{H}, \mathrm{Ar}-\mathrm{H}$ of coumarin ring and phenyl ring), $3.86\left(\mathrm{~s}, 3 \mathrm{H},-\mathrm{OCH}_{3}\right), 2.03\left(\mathrm{~s}, 3 \mathrm{H},-\mathrm{C}\left(\mathrm{C}_{3}\right)=\mathrm{N}-\right) .{ }^{13} \mathrm{C} \mathrm{NMR}\left(100 \mathrm{MHz}, \mathrm{DMSO}-d_{6}, \delta\right.$ ppm): 169.1, 163.9, 162.6, 160.4, 159.8, 155.9, 153.1, 153.0, 133.9, 132.6, 130.5, 130.5, 128.8, $128.8,128.5,128.5,128.1,127.6,127.6,126.6,125.6,123.9,118.2,116.3,115.5,115.5,115.1$, 114.8, 114.5, 114.5, 55.4, 4.6. MS: $\mathrm{m} / \mathrm{z} 539.16\left(\mathrm{M}^{+}\right)$. Anal. calcd. For $\mathrm{C}_{32} \mathrm{H}_{21} \mathrm{~N}_{5} \mathrm{O}_{4}, \mathrm{C}, 71.24 ; \mathrm{H}$, $3.92 ; \mathrm{N}, 12.98 \%$. Found: C, 71.18; H, 3.89; N, $12.95 \%$.

\section{2-oxo-1-((1-(2-oxo-2H-chromen-3-yl)ethylidene)amino)-4-phenyl-6-((3,4,5- trimethoxybenzylidene)amino)-1,2-dihydropyridine-3,5-dicarbonitrile (4n)}

Yield: $66 \%$, m.p. $229-233{ }^{\circ} \mathrm{C}$. IR $\left(\mathrm{KBr}, \mathrm{cm}^{-1}\right): 3129,3044$ (C-H stretching, aromatic ring), 2946 (C-H, $\left.\mathrm{CH}_{3}\right), 2220$ ( $-\mathrm{C} \equiv \mathrm{N}$ stretching, nitrile group), 1702 ( $>\mathrm{C}=\mathrm{O}$ stretching, coumarin ring), 1561, $1596(>\mathrm{C}=\mathrm{N}-,>\mathrm{C}=\mathrm{C}<$ stretching, aromatic ring), $1433(\mathrm{C}-\mathrm{H}$ bending, $-\mathrm{CH}=\mathrm{N}$ linkage $), 1251$ (C-O$\mathrm{C}$ coumarin ring), 1218, 1159 (C-O-C). ${ }^{1} \mathrm{H}$ NMR (300 MHz, DMSO- $d_{6}, \delta$ ppm): 9.25(s, 1H, Ar$\mathrm{CH}=\mathrm{N}-), 8.92(\mathrm{~s}, 1 \mathrm{H}, \mathrm{C} 4$ proton of coumarin), 7.12-7.78 $(\mathrm{m}, 11 \mathrm{H}, \mathrm{Ar}-\mathrm{H}$ of coumarin ring and phenyl ring), $3.83\left(\mathrm{~s}, 9 \mathrm{H},-\mathrm{OCH}_{3}\right), 2.08\left(\mathrm{~s}, 3 \mathrm{H},-\mathrm{C}\left(\mathrm{C}_{3}\right)=\mathrm{N}-\right) .{ }^{13} \mathrm{C}$ NMR $\left(100 \mathrm{MHz}, \mathrm{DMSO}-d_{6}, \delta\right.$ ppm): $169.7,163.5,160.2,159.7,155.3,153.5,153.5,153.5,153.2,141.6,133.5,132.5,128.8$, $128.8,128.5,128.5,128.2,128.0,127.7,127.7,125.6,123.5,118.4,116.6,115.9,115.9,115.5$, 114.6, 104.3, 104.3, 60.4, 56.5, 56.5, 4.6. MS: $\mathrm{m} / \mathrm{z} 599.18\left(\mathrm{M}^{+}\right)$. Anal. calcd. For $\mathrm{C}_{34} \mathrm{H}_{25} \mathrm{~N}_{5} \mathrm{O}_{6}, \mathrm{C}$, $68.11 ; \mathrm{H}, 4.20 ; \mathrm{N}, 11.68 \%$. Found: C, 68.02; H, 4.12; N, $11.72 \%$.

\section{6-((4-bromobenzylidene)amino)-2-oxo-1-((1-(2-oxo-2H-chromen-3-yl)ethylidene)amino)- 4-phenyl-1,2-dihydropyridine-3,5-dicarbonitrile (4o)}

Yield: $64 \%$, m.p. $208-212{ }^{\circ} \mathrm{C}$. IR $\left(\mathrm{KBr}, \mathrm{cm}^{-1}\right): 3140,3035$ (C-H stretching, aromatic ring), 2936 (C-H, $\left.\mathrm{CH}_{3}\right), 2207$ ( $\mathrm{C} \equiv \mathrm{N}$ stretching, nitrile group), 1719 ( $>\mathrm{C}=\mathrm{O}$ stretching, coumarin ring), 1552, $1593(>\mathrm{C}=\mathrm{N}-,>\mathrm{C}=\mathrm{C}<$ stretching, aromatic ring), $1424(\mathrm{C}-\mathrm{H}$ bending, $-\mathrm{CH}=\mathrm{N}$ linkage $), 1267$ (C-O$\mathrm{C}$ coumarin ring), 1084, $1012(\mathrm{C}-\mathrm{Br}) .{ }^{1} \mathrm{H}$ NMR $\left(300 \mathrm{MHz}, \mathrm{DMSO}-d_{6}, \delta \mathrm{ppm}\right): 9.11$ (s, $1 \mathrm{H}, \mathrm{Ar}-$ $\mathrm{CH}=\mathrm{N}-), 9.27(\mathrm{~s}, 1 \mathrm{H}, \mathrm{C} 4$ proton of coumarin), 7.14-7.85 (m, 13H, Ar-H of coumarin ring and phenyl ring), $2.08\left(\mathrm{~s}, 3 \mathrm{H},-\mathrm{C}\left(\mathrm{CH}_{3}\right)=\mathrm{N}-\right) .{ }^{13} \mathrm{C}$ NMR (100 MHz, DMSO- $d_{6}, \delta$ ppm): 169.2, 163.9, $160.3,159.7,155.8,153.4,153.2,133.7,132.3,132.2,131.6,131.6,128.9,128.9,128.7,128.7$, 
$128.4,128.4,128.2,127.8,127.8,125.4,125.4,123.5,118.3,116.4,115.7,115.7,115.5,114.6,4.5$. MS: m/z 587.06 $\left(\mathrm{M}^{+}\right)$. Anal. calcd. For $\mathrm{C}_{31} \mathrm{H}_{18} \mathrm{BrN}_{5} \mathrm{O}_{3}, \mathrm{C}, 63.28 ; \mathrm{H}, 3.08 ; \mathrm{N}, 11.90 \%$. Found: C, 63.36; H, 3.02; N, $11.86 \%$.

\section{SAR studies}

The structure-activity relationships (SAR) of compounds 4a-o were determined on the basis of results presented in Table 1. The substitution pattern on the coumarin based 2-pyridone ring system derivatives was carefully selected to confer different electronic environment of the molecules. From the activity data, compounds containing electron withdrawing group at para position lead to enhance bacterial activity. Compounds $\mathbf{4 g}\left(-4-\mathrm{NO}_{2}-\mathrm{C}_{6} \mathrm{H}_{4}\right), \mathbf{4 j}\left(-4-\mathrm{Cl}-\mathrm{C}_{6} \mathrm{H}_{4}\right)$ and $\mathbf{4 k}\left(-4-\mathrm{F}-\mathrm{C}_{6} \mathrm{H}_{4}\right)$ containing 4- $\mathrm{NO}_{2}, 4-\mathrm{Cl}$ and 4-F group showed highest inhibition at $\mathrm{MIC}=12.5-25 \mu \mathrm{g} \mathrm{ml}^{-1}$ against bacterial strain E. coli and $P$. aeruginosa. Same results observed in compounds $4 \mathbf{j}\left(-4-\mathrm{Cl}_{-} \mathrm{C}_{6} \mathrm{H}_{4}\right)$ and $4 \mathbf{k}\left(-4-\mathrm{F}-\mathrm{C}_{6} \mathrm{H}_{4}\right)$ showed highest inhibition at $\mathrm{MIC}=12.5-25 \mu \mathrm{g} \mathrm{m}{ }^{-1}$ against $S$. pyogenes and compound 4o $-4-\mathrm{Br}$ group showed highest inhibition at $\mathrm{MIC}=12.5 \mu \mathrm{g} \mathrm{ml}^{-1}$ against $P$. aeruginosa and $S$. aureus. On the basis of screening results, it has been observed that the compound containing electron releasing groups such as $-\mathrm{OH},-\mathrm{OCH}_{3}$ lead to enhance fungal activity. Compounds $\mathbf{4 d}(-4-$ $\left.\mathrm{OH}-\mathrm{C}_{6} \mathrm{H}_{4}\right), \mathbf{4} \mathbf{m}\left(-4-\mathrm{OCH}_{3}-\mathrm{C}_{6} \mathrm{H}_{4}\right)$ and $\mathbf{4 n}\left(-3,4,5-\left(\mathrm{OCH}_{3}\right)_{3}-\mathrm{C}_{6} \mathrm{H}_{2}\right)$ exhibited excellent inhibitory action against A.niger. While compounds $4 \mathbf{d}\left(-4-\mathrm{OH}-\mathrm{C}_{6} \mathrm{H}_{4}\right)$ and $\mathbf{4 m}\left(-4-\mathrm{OCH}_{3}-\mathrm{C}_{6} \mathrm{H}_{4}\right)$ also showed reasonably excellent inhibition against $C$. albicans and $A$. clavatus respectively. SAR studies revealed that the presence of electron withdrawing and electron releasing group in title compound increase the antibacterial and antifungal activity respectively.

\section{Conclusion}

New coumarin based 2-pyridone derivatives 4a-o were synthesized and screened for their antimicrobial activity. Compounds $4 \mathbf{g}\left(-4-\mathrm{NO}_{2}-\mathrm{C}_{6} \mathrm{H}_{4}\right), \mathbf{4 j}\left(-4-\mathrm{Cl}-\mathrm{C}_{6} \mathrm{H}_{4}\right), 4 \mathbf{k}\left(-4-\mathrm{F}-\mathrm{C}_{6} \mathrm{H}_{4}\right)$ and $\mathbf{4 o}(-4-$ Br- $\left.\mathrm{C}_{6} \mathrm{H}_{4}\right)$ exhibit outstanding antibacterial and compounds $\mathbf{4 d}\left(-4-\mathrm{OH}-\mathrm{C}_{6} \mathrm{H}_{4}\right), \mathbf{4 m}\left(-4-\mathrm{OCH}_{3}-\mathrm{C}_{6} \mathrm{H}_{4}\right)$ and 4n $\left(-3,4,5-\left(\mathrm{OCH}_{3}\right)_{3}-\mathrm{C}_{6} \mathrm{H}_{2}\right)$ exhibit antifungal properties. On the basis biological activity results can be concluded that above scaffolds would play an important role in the development of antimicrobial agents in future.

\section{Acknowledgements}

Authors are thankful to the University Grants Commission, New Delhi and Department of Science \& Technology, New Delhi for financial support under the NON-SAP and DST-FIST programs respectively.

\section{References}

[1] V.K. Vladimir, G.B. Alicia, Recent developments in the design and synthesis of hybrid molecules based on aminoquinoline ring and their antiplasmodial evaluation, Eur. J. Med. Chem. 44 (2009) 3091-3113.

[2] R.O. Kennedy, R.D. Thomas, Coumarins: Biology, Applications and Mode of Action, Wiley \& Sons, Chichester, 1997.

[3] M. Kawase, B. Varu, A. Shah, N. Motohashi, S. Tani, S. Saito, S. Debnath, S. Mahapatra, S.G. Dastidar, A.N. Chakrabarty, Antimicrobial activity of new coumarin derivatives, Arzneimittelforschung. 51 (2001) 67-71.

[4] K.B. Vyas, K.S. Mimavat, G.R. Jani, M.V. Hathi, Synthesis and antimicrobial activity of coumarin derivatives metal complex; An in vitro evaluation, Orbital. 1 (2009) 183-192.

[5] S. Bairagi, A. Bhosale, M.N. Deodhar, Design, Synthesis and Evaluation of Schiff's Bases of 4Chloro-3-coumarin aldehyde as Antimicrobial Agents, E-J. Chem. 6 (2009) 759-762. 
[6] S.E. Lopez, M.E. Rosales, C.E. Canelon, E.A. Valverode, R.C. Narvaez, J.E. Charris, F.A. Giannini, R.D. Enriz, M. Carrasco, S. Zacchino, Synthesis and preliminary cytotoxic and antifungal evaluation of some 6-n,n-dialkyl 2-aryl-4(3h)-quinazolinone derivatives, Hetrocycl. Commun. 7 (2000) 473-480.

[7] K.M. Khan, S.S. Zafar, Z.K. Muhammad, Zia-Ullah, C.M. Iqbal, Atta-ur-Rahman, P. Shahnaz, H.C. Zahid, T.S. Claudiu, Synthesis of coumarin derivatives with cytotoxic, antibacterial and antifungal activity, Enzyme Inhib. Med. Chem. 19 (2004) 373-379.

[8] Y. Bansal, P. Sethi, G. Bansal, Coumarin: a potential nucleus for anti-inflammatory molecules, Med. Chem. Res. 22 (2013) 3049-3060.

[9] K.C. Fylaktakidou, D.J. Hadjipavlou-Litina, K.E. Litinas, D.N. Nicolaides, Natural and synthetic coumarin derivatives with anti-inflammatory/antioxidant activities, Curr. Pharm. Des. 10 (2004) 3813-3833.

[10] M.A. Musa, J.S. Cooperwood, M.O.F. Khan, A review of coumarin derivatives in pharmacotherapy of breast cancer, Curr. Med. Chem. 15 (2008) 2664-2679.

[11] C. Kontogiorgis, A. Detsi, D. Hadjipavlou-Litina, Coumarin-based drugs: a patent review, Expert Opin. Ther. Patents. 22 (2012) 437-454.

[12] P.O. Patil, S.B. Bari, S.D. Firke, P.K. Deshmukh, S.T. Donda, D.A. Patil, A comprehensive review on synthesis and designing aspects of coumarin derivatives as monoamine oxidase inhibitors for depression and Alzheimer's disease, Bioorg. Med. Chem. 21 (2013) 2434-2450.

[13] S.V. Dekic, V.S. Dekic, B.R. Dekic, M.S. Dekic, Synthesis of new condensed and cyclized coumarin derivatives, Chemical Papers. 61 (2007) 233-235.

[14] W.B. Choi, I.N. Houpis, H.R.O. Churchill, A. Molina, J.E. Lynch, R.P. Volante, P.J. Reider, A.O. King, A practical synthesis of the 5-chloromethyl-furo[2,3-b]pyridine pharmacophores, Tetrahedron Lett. 36 (1995) 4571-4574.

[15] M. Bhupathy, D.A. Conlon, K.M. Wells, J.R. Nelson, P.J. Reider, K. Rossen, J.W. Sager, R.P. Volante, B.D. Dorsey, J.M. Hoffman, S.A. Joseph, S.L.J. McDaniel, A practical synthesis of 5-(chloromethyl)furo[2,3-b]pyridine, a key intermediate for the HIV protease inhibitor, Heterocycl. Chem. 32 (1995) 1283-1287.

[16] C.O. Kappe, T. Kappe, Synthesis of substituted 3-pyridinecarbonitriles with potential biological activity, Monatsh. Chem. 120 (1989) 1095-1100.

[17] G. Ozturk, D.D. Erol, T. Uzbay, M.D. Aytemir, Synthesis of 4(1H)-pyridinone derivatives and investigation of analgesic and anti-inflammatory activities, Farmaco. 56 (2001) 251-256.

[18] A. Abadi, O. Al-Deeb, A. Al-Afify, H. El-Kashef, Synthesis of 4-alkyl (aryl)-6-aryl-3-cyano2( $1 H$ )-pyridinones and their 2-imino isosteres as nonsteroidal cardiotonic agents, Farmaco. 54 (1999) 195-201.

[19] P. Storck, A. Aubertin, D.S. Grierson, Tosylation/mesylation of 4-hydroxy-3-nitro-2pyridinones as an activation step in the construction of dihydropyrido [3,4b] benzo [f] [1,4]thiazepin-1-one based anti-HIV agents, Tetrahedron Lett. 46 (2005) 2919-2922.

[20] A. Evidente, M. Fiore, G. Bruno, L. Sparapano, A. Motta, Chemical and biological characterisation of sapinopyridione, a phytotoxic 3,3,6-trisubstituted-2,4-pyridione produced by Sphaeropsis sapinea, a toxigenic pathogen of native and exotic conifers and its derivatives, Phytochemistry. 67 (2006) 1019-1028.

[21] M.T. Cocco, C. Congiu, V. Onnis, New bis(pyridyl)methane derivatives from 4-hydroxy-2pyridones: synthesis and antitumoral activity, Eur. J. Med. Chem. 38 (2003) 37-47. 
[22] Q. Li, L.A. Mitscher, L.L. Shen, The 2-pyridone antibacterial agents: bacterial topoisomerase inhibitors, Med. Res. Rev. 20 (2000) 231-293.

[23] L.A. Mitscher, Bacterial topoisomerase inhibitors: quinolone and pyridone antibacterial agents, Chem. Rev. 105 (2005) 559-592.

[24] N.C. Desai, N. Bhatt, A. Dodiya, T. Karkar, B. Patel, M. Bhatt, Synthesis, characterization and antimicrobial screening of thiazole based 1,3,4-oxadiazoles heterocycles, Res. Chem. Intermed. (2015) published ahead of print; DOI: 10.1007/s11164-015-2196-X.

[25] N.C. Desai, N.R. Shihory, G.M. Kotadiya, P. Desai, Synthesis, antibacterial and antitubercular activities of benzimidazole bearing substituted 2-pyridone motifs, Eur. J. Med. Chem. 82 (2014) 480-489.

[26] N.C. Desai, N. Shihory, M. Bhatt, B. Patel, T. Karkar, Studies on antimicrobial evaluation of some 1-((1-(1h-benzo[d]imidazol-2-yl)ethylidene)amino)-6-((arylidene) amino)-2-oxo-4phenyl-1,2-dihydropyridine-3,5-dicarbonitriles, Synth. Commun. 45 (2015) 2701-2711.

[27] National committee for clinical laboratory, Standards Methods for Dilution Antimicrobial Susceptibility Tests for Bacteria that Grow Aerobically approved standard, third ed. NCCLS Publication M7-A3, Villanova, PA, 1993.

[28] National committee for clinical laboratory standards, Reference Method for Broth Dilution Antifungal Testing of Yeasts, Proposed Standard, NCCLS Document M27-P; Villanova, PA, 1992.

[29] N.C. Desai, V.V. Joshi, K.M. Rajpara, H.V. Vaghani, H.M. Satodiya, Facile synthesis of novel fluorine containing pyrazolebased thiazole derivatives and evaluation of antimicrobial activity, J. Fluorine Chem. 142 (2012) 67-78.

[30] N.C. Desai, K.M. Rajpara, V.V. Joshi, Synthesis and characterization of some new quinoline based derivatives endowed with broad spectrum antimicrobial potency, Bioorg. Med. Chem. Lett. 22 (2012) 6871- 6875. 